\title{
Acoustic and Thermo-Dynamical Investigation of 2-Amino-5-Nitrothiazole- DMSO-Water System at Different Temperatures and Concentration
}

Thakare $\mathrm{AR}^{1^{*}}$, Dongapure $\mathrm{AC}^{1}$ and Naik $\mathrm{AB}^{2}$

${ }^{1}$ Shankarlal Agrawal Science College, Salekasa, Gondia District, Maharashtra, India

${ }^{2}$ Department of Chemical Technology, Sant Gadge Baba Amravati University, Amravati, Maharashtra, India

\begin{abstract}
Thiazole is Sulphur and nitrogen containing heterocyclic compounds represents an important family of medicines in the pharmaceutical chemistry having vast biological applications. Due to the presence of hetero atoms in 2-amino-5nitrothiazole there should be various types of interaction, by undertaking these interactions, ultrasonic study of 2-amino5-nitrothiazole in 70:30 (v/v) DMSO-Water mixture at different concentration and temperature is carried out. Speed of sound and denseness of ternary mixture of 2-amino-5-nitrothiazole - 70:30 (v/v) DMSO-water systems has been measured at various temperature $(303.15 \mathrm{~K}, 308.15 \mathrm{~K}, 313.15 \mathrm{~K}$ and $318.15 \mathrm{~K})$ and concentrations, to get idea regarding the solute- solvent and solvent - solvent interaction. In present investigation ultrasonic parameters i.e., specific acoustic impedance, relative association intermolecular free length, adiabatic compressibility, and sound speed number has been put forward to say about types of molecular interaction present in the aqueous 70:30 (v/v) DMSO-water solution of various temperatures and concentrations. Strength of molecular interactions was studied with comparisons to change in temperature and change in concentration. The correlation observed represents interpreted in the form of solute-solvent and solvent-solvent interactions at given temperature variation and concentration variations.
\end{abstract}

Keywords: Heterocyclic; Therapeutic chemistry; Ultrasonic technique; Thiazole

\section{Introduction}

Heterocyclic chemistry is the important branch of the organic chemistry and have great importance biologically and industrially [1]. Heterocyclic molecules have great application in therapeutic chemistry and play key role in development of most of the therapeutic drugs [2]. Hetero atoms containing cyclic compound have wide range of physiological properties i.e., antibacterial, anti-inflammatory, muscle relaxant, lipid peroxidation inhibitor ant tubercular and insecticidal [38]. In present investigation the heterocyclic compounds use has great importance in life process [9]. Present study is the part of our recent work [9-11] on ultrasonic study of derivatives of thiazole. Ultrasonic technique is powerful tool for the studies of physicochemical properties of a system. Although DMSO fluid solution has been mostly studied however, survey of literature indicate that no acoustical and thermo dynamical investigation have been taken into consideration of 2-amino-5-nitrothiazole-DMSO system. Acoustical parameters such as speed of sound, denseness, acoustical impedance, relative association, etc. of 2-amino-5-nitrothiazole - 70:30 (V/V) DMSO-Water in aqueous solution at given temperatures and various concentrations measured in this study was lacking and hence we attempt to study acoustical parameters such as speed of sound, denseness, acoustical impedance, relative association etc of 2-amino-5-nitrothiazole - 70:30 (V/V) DMSOWater in aqueous solution at various temperature and concentrations so as to know effect of temperatures and concentrations on ultrasonic parameters. The great importance of 2-amino-5-nitrothiazole in different field of science makes our interest in the measurements and interpretation of their acoustical properties in order to understand the solute - solvent and solvent - solvent interaction at various temperature and concentrations.

\section{Materials and Experimental}

The solvent DMSO of anal grade used without purification while, water used in present study was double distilled using $\mathrm{KMnO}_{4}$ and $\mathrm{NaOH}$. The synthesis grade 2-amino-5-nitrothiazole (Figure 1) (HiMEDIA, minimum wt 97\%), use as solute. The experimental solutions were prepared by dissolving 2-amono-5-nitrothiazole van accurate amount in an organic solute-aqueous mixture in standard flask having airtight caps and the mass were measured on high precision digital balance (Adair Datta having accuracy up to $\pm 0.01 \mathrm{mg}$ ). The speed of sound of pure component and their mixtures were measured by ultrasonic instrument (Mittal enterprises, model F-81s) at $2 \mathrm{MHz}$ having frequency tolerance $\pm 0.03 \%$. The denseness of DMSO and ligand solutions was guarded by using digital density meter (Anton Paar DMA 35).

\section{Theory}

Given acoustical parameters are calculated by using various equations.

Ultrasonic velocity $(u)=v \lambda$

Where $\mathrm{u}=$ ultrasonic velocity, $\lambda=$ wavelength.

Isentropic compressibility $\left(\beta_{\mathrm{s}}\right)=1 / \rho \mathrm{u}^{2}$

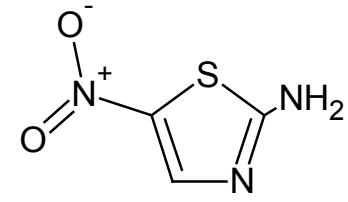

Figure 1: Structure of 2-amono-5-nitrothiazole.

*Corresponding author: Thakare AR, Shankarlal Agrawal Science College, Salekasa, Gondia District-441 916, Maharashtra, India, Tel: +9970787768; E-mail: avinashrthakare@yahoo.com

Received December 26, 2016; Accepted January 19, 2017; Published January 23, 2017

Citation: Thakare AR, Dongapure AC, Naik AB (2017) Acoustic and Thermo-Dynamical Investigation of 2-Amino-5-Nitrothiazole-DMSO-Water System at Different Temperatures and Concentration. Med Chem (Los Angeles) 7: 745-749. doi: 10.4172/2161-0444.1000424

Copyright: (c) 2017 Thakare AR, et al. This is an open-access article distributed under the terms of the Creative Commons Attribution License, which permits unrestricted use, distribution, and reproduction in any medium, provided the original author and source are credited. 
Citation: Thakare AR, Dongapure AC, Naik AB (2017) Acoustic and Thermo-Dynamical Investigation of 2-Amino-5-Nitrothiazole-DMSO-Water System at Different Temperatures and Concentration. Med Chem (Los Angeles) 7: 745-749. doi: 10.4172/2161-0444.1000424

Where $\rho=$ density $u=$ speed of sound.

Intermolecular free length $\left(L_{\mathrm{f}}\right)=\mathrm{K} \beta_{\mathrm{s}}^{1 / 2}$

Where $\mathrm{K}=J$ acobson constant [13]

Acoustic impedance $(Z)=u \rho$

Relative association $\left(\mathrm{R}_{\mathrm{A}}\right)=\left(\rho / \rho_{\mathrm{o}}\right)\left(u_{\mathrm{o}} / u\right)^{1 / 3}$

Where $\rho_{\mathrm{o}}=$ density of solvent, $u_{\mathrm{o}}=$ velocity of solvent. Also the sound velocity number is calculated from following equation

Sound velocity number $[\mathrm{U}]=\mathrm{u}-\mathrm{u}_{\mathrm{o}} / \mathrm{u}_{\mathrm{o}} \mathrm{c}$

$[\mathrm{U}]=$ sound velocity number, $\mathrm{c}=$ concentration of the solute.

\section{Results and Discussion}

The practically taken values of speed of sound and density for the pure DMSO solvent and mixtures at $(303.15 \mathrm{~K}, 308.15 \mathrm{~K}, 313.15 \mathrm{~K}$ and $318.5 \mathrm{~K})$ are given in Table 1 . From the practically determined data, various thermo dynamical and acoustical parameters such as intermolecular free length, relative association isentropic compressibility, sound velocity number and acoustic impedance have been computed and are given in Table 1.

The observation of Figure 2 indicates that the value of denseness

\begin{tabular}{|c|c|c|c|c|c|c|c|}
\hline C $\mathrm{mol} / \mathrm{lit}$ & $\mathrm{u}, \mathrm{m} \mathrm{s}^{-1}$ & $\rho, \mathbf{k g ~ m}^{-3}$ & $\beta s \times 10^{-10}, m^{2} . N^{-1}$ & $L_{f}, A_{0}$ & $Z \times 10^{-5} \mathrm{~kg} \cdot \mathrm{m}^{2} \mathrm{~s}^{-1}$ & $\mathbf{R}_{\mathrm{A}}$ & {$[\mathrm{U}], \mathrm{kg} \mathrm{mol}^{-1}$} \\
\hline 0.00 & 1470 & 1.095 & 4.2262 & $\begin{array}{c}303.15 K \\
0.4282\end{array}$ & 16.096 & - & - \\
\hline 0.01 & 1468 & 1.098 & 4.2261 & 0.4282 & 16.118 & 0.998652 & -0.13605 \\
\hline 0.02 & 1467 & 1.105 & 4.2051 & 0.4271 & 16.21 & 1.002967 & -0.10204 \\
\hline 0.03 & 1463 & 1.112 & 4.2015 & 0.4269 & 16.268 & 1.001087 & -0.15873 \\
\hline 0.04 & 1460 & 1.119 & 4.1924 & 0.4265 & 16.337 & 1.001204 & -0.17006 \\
\hline 0.05 & 1457 & 1.122 & 4.1984 & 0.4268 & 16.347 & 0.997712 & -0.17687 \\
\hline 0.06 & 1454 & 1.128 & 4.1933 & 0.4265 & 16.401 & 0.996865 & -0.18140 \\
\hline 0.07 & 1451 & 1.131 & 4.1995 & 0.4268 & 16.41 & 0.993342 & -0.18462 \\
\hline 0.08 & 1446 & 1.139 & 4.1984 & 0.4268 & 16.469 & 0.990062 & -0.20408 \\
\hline 0.09 & 1442 & 1.144 & 4.2038 & 0.4270 & 16.496 & 0.986179 & -0.21164 \\
\hline \multirow[t]{2}{*}{0.10} & 1436 & 1.153 & 4.2059 & 0.4271 & 16.557 & 0.981582 & -0.23129 \\
\hline & & & & $308.15 K$ & & & \\
\hline 0.00 & 1466 & 1.072 & 4.3404 & 0.4339 & 15.715 & - & - \\
\hline 0.01 & 1463 & 1.078 & 4.3340 & 0.4336 & 15.771 & 0.999436 & -0.20463 \\
\hline 0.02 & 1458 & 1.085 & 4.3356 & 0.4337 & 15.819 & 0.995648 & -0.27285 \\
\hline 0.03 & 1453 & 1.096 & 4.3217 & 0.4330 & 15.924 & 0.995430 & -0.29558 \\
\hline 0.04 & 1449 & 1.102 & 4.3219 & 0.4330 & 15.967 & 0.992636 & -0.28990 \\
\hline 0.05 & 1445 & 1.105 & 4.3341 & 0.4336 & 15.967 & 0.987118 & -0.28649 \\
\hline 0.06 & 1440 & 1.110 & 4.3446 & 0.4341 & 15.984 & 0.981327 & -0.29558 \\
\hline 0.07 & 1435 & 1.116 & 4.3514 & 0.4345 & 16.014 & 0.976390 & -0.30208 \\
\hline 0.08 & 1431 & 1.123 & 4.3485 & 0.4343 & 16.070 & 0.974321 & -0.29843 \\
\hline 0.09 & 1424 & 1.131 & 4.3603 & 0.4349 & 16.105 & 0.966932 & -0.31832 \\
\hline \multirow[t]{2}{*}{0.10} & 1417 & 1.142 & 4.3610 & 0.4349 & 16.182 & 0.962009 & -0.33424 \\
\hline & & & & $313.15 K$ & & & \\
\hline 0.00 & 1463 & 1.065 & 4.3869 & 0.4362 & 15.580 & - & - \\
\hline 0.01 & 1459 & 1.073 & 4.3781 & 0.4358 & 15.655 & 0.999270 & -0.27341 \\
\hline 0.02 & 1453 & 1.078 & 4.3939 & 0.4366 & 15.663 & 0.991592 & -0.34176 \\
\hline 0.03 & 1448 & 1.087 & 4.3876 & 0.4363 & 15.739 & 0.989584 & -0.34176 \\
\hline 0.04 & 1441 & 1.093 & 4.4060 & 0.4372 & 15.750 & 0.980685 & -0.37593 \\
\hline 0.05 & 1436 & 1.101 & 4.4045 & 0.4371 & 15.810 & 0.977615 & -0.36910 \\
\hline 0.06 & 1430 & 1.107 & 4.4175 & 0.4378 & 15.830 & 0.970673 & -0.37593 \\
\hline 0.07 & 1427 & 1.113 & 4.4122 & 0.4375 & 15.882 & 0.969805 & -0.35152 \\
\hline 0.08 & 1421 & 1.118 & 4.4296 & 0.4384 & 15.886 & 0.961926 & -0.35885 \\
\hline 0.09 & 1415 & 1.125 & 4.4395 & 0.4388 & 15.918 & 0.955739 & -0.36454 \\
\hline 0.10 & 1409 & 1.129 & 4.4615 & 0.4399 & 15.907 & 0.946988 & -0.36910 \\
\hline 0.00 & 1458 & 1.058 & 4.4463 & $\begin{array}{c}\mathbf{3 1 8 . 1 5 K} \\
0.4392\end{array}$ & 15.425 & & \\
\hline 0.01 & 1453 & 1.062 & 4.4601 & 0.4399 & 15.430 & 0.993489 & -0.34293 \\
\hline 0.02 & 1449 & 1.069 & 4.4553 & 0.4396 & 15.489 & 0.991801 & -0.30864 \\
\hline 0.03 & 1441 & 1.074 & 4.4840 & 0.4410 & 15.476 & 0.980027 & -0.38866 \\
\hline 0.04 & 1437 & 1.077 & 4.4964 & 0.4416 & 15.476 & 0.974603 & -0.36008 \\
\hline 0.05 & 1432 & 1.085 & 4.4945 & 0.4416 & 15.537 & 0.971629 & -0.35665 \\
\hline 0.06 & 1424 & 1.091 & 4.5201 & 0.4428 & 15.535 & 0.960719 & -0.38866 \\
\hline 0.07 & 1417 & 1.098 & 4.5358 & 0.4436 & 15.558 & 0.952694 & -0.40172 \\
\hline 0.08 & 1411 & 1.108 & 4.5332 & 0.4434 & 15.638 & 0.949211 & -0.40294 \\
\hline 0.09 & 1407 & 1.116 & 4.5263 & 0.4431 & 15.792 & 0.947956 & -0.38866 \\
\hline 0.10 & 1402 & 1.123 & 4.5302 & 0.4433 & 15.744 & 0.943769 & -0.38408 \\
\hline
\end{tabular}

Table 1: Experimental values of various acoustical and thermo dynamical parameter for 2-amino-5-nitrothiazole-70:30 DMSO-water mixtures at different concentration and 303.15K, 308.15K, 313.15K and 318.15K. 
Citation: Thakare AR, Dongapure AC, Naik AB (2017) Acoustic and Thermo-Dynamical Investigation of 2-Amino-5-Nitrothiazole-DMSO-Water System at Different Temperatures and Concentration. Med Chem (Los Angeles) 7: 745-749. doi: 10.4172/2161-0444.1000424

increases not only due to increase in temperature but also increased in the concentration of 2-amino-5-nitrothiazole. Existence of solute - solvent molecular interaction has been proved from the increased values of density. The increased density is concern to the presence of strong intermolecular attraction such as dipole-dipole attraction and hydrogen bonding. Addition of the organic solute to the solvent not only allows stronger association in between solvent and solute molecules but also allows closer approach of solute and solvent molecules. This tends to decrease in the volume and an increase in the density of the solution $[12,13]$. From Figure 3 it observe that at high temperature heteromolecular and homomolecular clusters breaks which leads to the decrease in values of speed of sound with increase in temperature [14]. It has been observe that the change in speed of sound depends on the structure and properties of solute. Therefore, the solutes which increase the ultrasonic velocity are structure maker while those decreases the ultrasonic velocities are structure breakers [13]. The decrease in ultrasonic velocity shows that the interaction between solute and solvent is becoming less dominant. This is due to the replacement of strong intermolecular attraction between solvent molecules by weaker intermolecular interactions. This shows that the solvent-solvent interaction substitutes by solute-solvent interaction. When ultra sound wave falls on the solution the perturbation of molecules takes place. The reason is medium has elastic properties and hence equilibrium position regained by perturbed molecules [15]. When a solute is added to a solvent at that time molecules of solute attract certain molecules of solvent toward them which is known as compression. Every solvent has a limit for compression and is known as limiting compressibility. The solute-solvent interaction is supports from the increased values of isentropic compressibility due to increase of temperature and concentration which is evident from Figure 4. When solute mixed with the solvent the variation in speed of sound is dependent on intermolecular free length. Erying and Kincaid model of sound propagation suggest that [16], values of free length decrease with increase in ultrasonic velocity and vice versa. For the determination of variation in speed of ultrasound in liquids and their solutions a most important thing is Intermolecular free length [17]. The distance between the surfaces of the neighboring molecules known as Intermolecular free length which indicates a significant interaction between solvent-solvent as well as solute-solvent molecules [18]. Hence, it is also a good tool to investigate the molecular interactions in the binary solvent mixture, from Figure 5 it is also clear that as temperature increases intermolecular

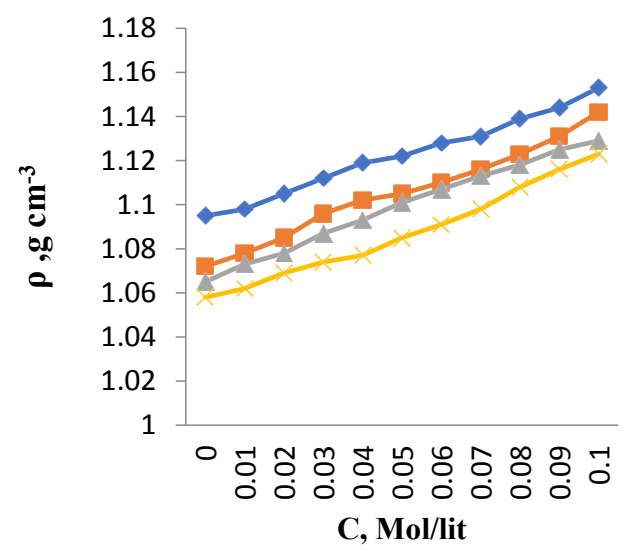

Figure 2: Density ( $\rho)$ plotted against concentration of 2-amino-5nitrothiazole-70:30 DMSO-Water solutions at temperatures $303.15 \mathrm{~K}(\bullet)$, 308.15K (ש), 313.15K ( $\mathbf{\Delta}), 318.15 \mathrm{~K}(\times)$.

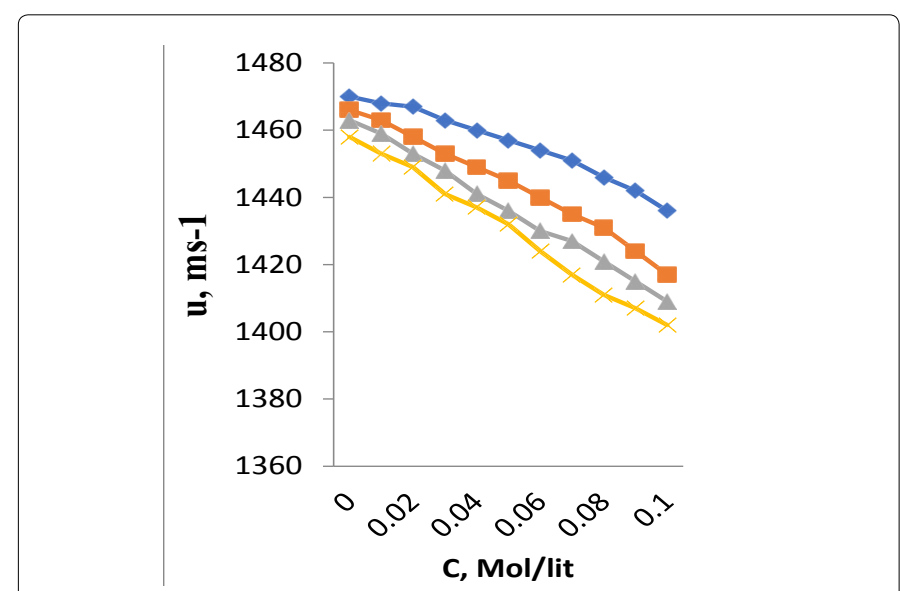

Figure 3: Ultrasonic velocity (u) plotted against concentration of 2-amino5-nitrothiazole-70:30 DMSO-Water solutions at temperatures $303.15 \mathrm{~K}(\bullet)$, 308.15K (घ), 313.15K ( $\mathbf{\Delta})$, 318.15K (x).

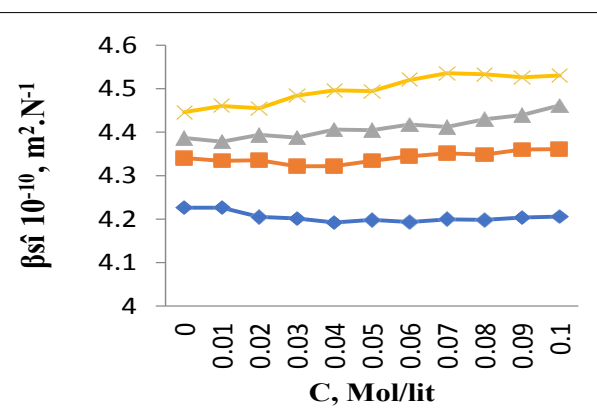

Figure 4: Isentropic compressibility $(\beta s)$ plotted against concentration of 2-amino-5-nitrothiazole-70:30 DMSO-Water solutions at temperatures 303.15K (•), 308.15K (匹), 313.15K ( $\mathbf{\Delta})$, 318.15K (×).

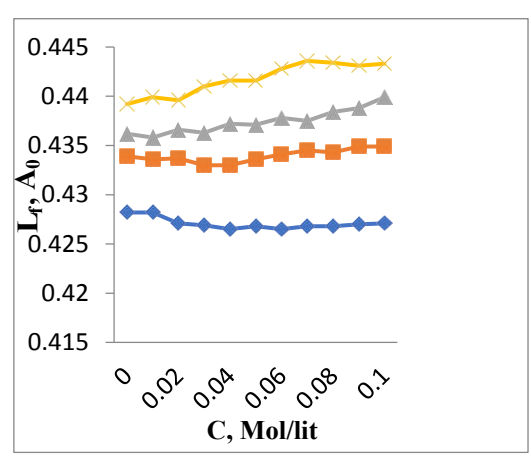

Figure 5: Linear free length (Lf) plotted against concentration of 2-amino5-nitrothiazole-70:30 DMSO-Water solutions at temperatures 303.15K ( $\bullet$ ), 308.15K (घ), 313.15K ( $\mathbf{\Delta})$, 318.15K (×).

free length also increases. When the ultrasonic wave propagate through a solution, some part of it of ultrasonic wave gets reflected by the ion [19] and remaining part travels through the medium it means ions restricts free propagation of sound wave, this restriction of sound wave known as acoustic impedance $(Z)$. If the temperature increases, ultrasonic velocity decreases and value of acoustic impedance ( $\mathrm{z}$ ) decreases [17] this is because acoustic impedance $(Z)$ is the product of speed of ultrasound $(u)$ and denseness $(\rho)$ indicates from Figure 6. From Figure 7 it is also seen that value of 
Citation: Thakare AR, Dongapure AC, Naik AB (2017) Acoustic and Thermo-Dynamical Investigation of 2-Amino-5-Nitrothiazole-DMSO-Water System at Different Temperatures and Concentration. Med Chem (Los Angeles) 7: 745-749. doi: 10.4172/2161-0444.1000424

relative association $\left(R_{A}\right)$ increases along with temperature. The value of $R_{A}$ depends on either the breaking up of the solvent molecules on addition of solute molecules in solvent at certain temperature or the solvation of ions that are present $[20,21]$. In general sound velocity number increases with increase in concentration of solute [22] and increase in temperature; however in present investigation it is decreases with increase of concentration of solute and increases with increase of temperature (Figure 8) which also suggest the strong intermolecular interaction in the solution.

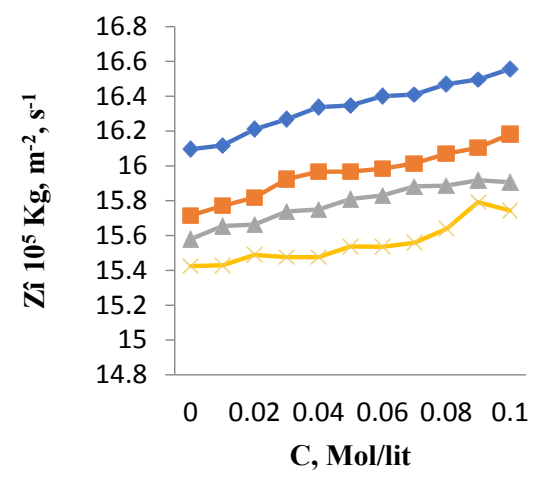

Figure 6: Acoustic impedance(z) plotted against concentration of 2-amino5-nitrothiazole-70:30 DMSO-Water solutions at temperatures 303.15K ( $\bullet$ ), 308.15K (घ), 313.15K ( $\mathbf{\Delta}), 318.15 \mathrm{~K}(\times)$.

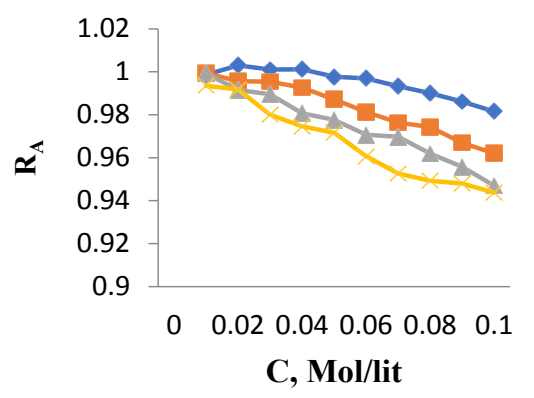

Figure 7: Relative association (RA) plotted against concentration of 2-amino5-nitrothiazole-70:30 DMSO-Water solutions at temperatures $303.15 \mathrm{~K}(\star)$, 308.15K (घ), 313.15K ( $\mathbf{\Delta}), 318.15 \mathrm{~K}(\times)$.

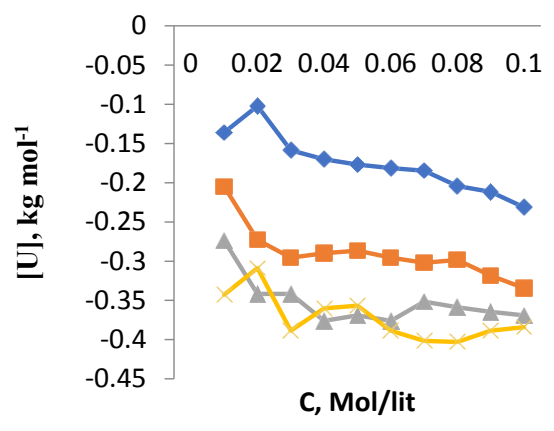

Figure 8: Sound velocity number [U] plotted against concentration of 2-amino-5-nitrothiazole-70:30 DMSO-Water solutions at temperatures 303.15K (•), 308.15K (•), 313.15K ( $\Delta)$, 318.15K (×).

\section{Conclusion}

The acoustical method is powerful tool for investigating physicochemical properties and presence molecular interaction in ternary mixtures. The result indicates that the denseness and speed of ultrasound of 2-amino-5-nitrothiazole-70:30 DMSO-water mixture increases and decreases respectively with increase in concentration and temperature. The existence of molecular interactions proves from linear plots of respective acoustical parameters. These results clearly indicate scope for further studies on effect of temperature and concentration on molecular interactions. These results also give the scope for investigating acoustical parameters of various substituted thiazoles as a ligand with aqueous metal solution [23].

\section{References}

1. Gupta V, Kant V (2013) A Review on Biological Activity of Imidazole and Thiazole Moieties and their Derivatives. Sci. International 1: 253-260.

2. Shah $P$ (2014). Studies of novel thiazole-imidazole combined molecule. Octa Journal of Environmental Research 2: 48-52.

3. Shah $P(2012)$ Synthesis and biological activity of novel imidazole. Current Trends in Biotechnology and Chemical Research 2: 45-51A.

4. Mittal A (2009) Synthetic Nitroimidazoles: Biological Activities and Mutagenicity Relationships. Sci. Pharm 77: 497-520.

5. Nagalakshmi G (2008) Synthesis, Antimicrobial and Antiinflammatory Activity of 2,5-Disubstituted-1,3,4-oxadiazoles. Indian J Pharm Sci 70: 49-55.

6. Nekrasov DD (2001) Biological Activity of 5-and 6-Membered Azaheterocycles and Their Synthesis from 5-Aryl-2, 3-Dihydrofuran-2, 3-diones.(Review) Chemistry of Heterocyclic Compounds 37: 263-275.

7. Sperry JB1, Wright DL (2005) Furans, thiophenes and related heterocycles in drug discovery. Curr Opin Drug Discov Devel 8: 723-740.

8. Polshettiwar V, Varma RS (2008) Greener and expeditious synthesis of bioactive heterocycles using microwave irradiation. Pure and Applied Chemistry 80: 777-790.

9. Thakare AR, Naik AB (2016) Study of molecular interactions of 2-amino-5nitrothiazole in NNDMF, acetonitrile, and ethanol using acoustical parameters. Cogent chemistry 2: 1242368.

10. Akwuobu CA, Chah KF, Oboegbulem SI, Rabo JS (2016) Pathogenicity of local isolates of Mycoplasma ovipneumoniae and Mycoplasma arginini in experimental West African Dwarf goats. Journal of Advanced Veterinary and Animal Research 3: 242-251.

11. Thakare A, Dongapure A, Rathod V, Naik A (2015) Acoustical Study of Substituted Thiazoles in Nndmf - Water Mixtures at Different Temperatures. IJRBAT 6: 347-350.

12. Syal VK, Patial BS, Chauhan S (1999) Ultrasonic velocity, viscosity and density studies in binary mixtures of dimethyl formamide and ethylmethylketone at different temperatures IJPAP 37: 366-370.

13. Aswar AS, Choudhary DS (2013) Densities and ultrasonic speed of 2-hydroxy5-methyl-3-nitro acetophenone in $\mathrm{N}, \mathrm{N}$-dimethylformamide at different temperatures. Bulletin of the Chemical Society of Ethiopia 27: 155-160.

14. Rambabu C (2013) Thermodynamic and acoustic study on molecular interactions in certain binary liquid systems involving ethyl benzoate. Journal of Thermodynamics ID 285796.

15. Tiwari S, Kusmariya BS, Tiwari A, Pathak V, Mishra AP (2015) Acoustical and viscometric studies of buspirone hydrochloride with cobalt (II) and copper (II) ions in aqueous medium. Journal of Taibah University for Science 11: 002.

16. Kincaid JF, Eyring H (1939) The Liquid State. Journal of Physical Chemistry 43: $37-47$.

17. Landge M, Badade S, Kendre B (2013) Density, ultrasonic velocity and viscosity measurements of Gluc Glucose-Alcohol-Water mixtures at various temperatures. International Journal of Research in Chemistry and environment 3: 348-352.

18. Kharkale S, Bhaskar C, Agarwal P, Paliwal P (2013) Int. J. of Emerging Techs in Computational and App Scis. 5: 327-329.

19. Yadav P, Kumar M. Yadav (2014) Study of molecular interaction in binary liquid 
Citation: Thakare AR, Dongapure AC, Naik AB (2017) Acoustic and Thermo-Dynamical Investigation of 2-Amino-5-Nitrothiazole-DMSO-Water System at Different Temperatures and Concentration. Med Chem (Los Angeles) 7: 745-749. doi: 10.4172/2161-0444.1000424

mixtures of ethyl acetoacetate with chloroform and dimethylsulphoxide using excess acoustic parameters and spectroscopic methods. Taylor\& Francis, Phys and Chem. of Liqs. 52: 331-341.

20. Ambomase SS, Tripathy S, Tripathy M, Dash UN (2011) Studies on waterpolymer interactions inthe presence of aceclofenac at $298.15 \mathrm{~K}$. Journal of Chemistry 8: 63-70.

21. Meshram B, Agrawal P, Chandak H, Chapke U (2013) A study of acoustical behaviour of paracetamol in $70 \%$ methanol at various temperature. International
Journal of Emerging Technologies in Computational and Applied Sciences 5: 369-373.

22. Chauhan S (2016) Study of acoustic parameters of proline in lecithin-ethanol mixture at varying temperature. Indian Journal of Pure \& Applied Physics (IJPAP) 51: 531-541.

23. Thakare AR, Naik AB (2016) Study of Molecular Interactions in Aqueous 2-Amino-5-Nitrothiazole-NiCl2 Solution at Different Temperatures and Concentrations. ChemXpress 9: 110. 